\title{
Adaptive-sampling near-Doppler-limited terahertz dual-comb spectroscopy with a free-running single-cavity fiber laser
}

\author{
Jie Chen, ${ }^{\text {a,b }}$ Kazuki Nitta, ${ }^{\mathrm{b}, \mathrm{c}}$ Xin Zhao, ${ }^{\mathrm{a}}$ Takahiko Mizuno, ${ }^{\mathrm{c}, \mathrm{de}}$ Takeo Minamikawa, ${ }^{\mathrm{c}, \mathrm{d}, \mathrm{e}, \mathrm{f}}$ Francis Hindle, ${ }^{\mathrm{g}}$ Zheng Zheng, ${ }^{\mathrm{a}, \mathrm{h}, \boldsymbol{}}$ \\ and Takeshi Yasui ${ }^{c, d, e, f, *}$ \\ aBeihang University, School of Electronic and Information Engineering, Beijing, China \\ ${ }^{\text {DT} T o k u s h i m a ~ U n i v e r s i t y, ~ G r a d u a t e ~ S c h o o l ~ o f ~ A d v a n c e d ~ T e c h n o l o g y ~ a n d ~ S c i e n c e, ~ T o k u s h i m a, ~ J a p a n ~}$ \\ 'JST, ERATO MINOSHIMA Intelligent Optical Synthesizer, Tokushima, Japan \\ dTokushima University, Institute of Post-LED Photonics, Tokushima, Japan \\ ${ }^{\text {eT} T o k u s h i m a ~ U n i v e r s i t y, ~ G r a d u a t e ~ S c h o o l ~ o f ~ T e c h n o l o g y, ~ I n d u s t r i a l ~ a n d ~ S o c i a l ~ S c i e n c e s, ~ T o k u s h i m a, ~ J a p a n ~}$ \\ 'Tokushima University, Research Cluster on "Multi-scale Vibrational Microscopy for Comprehensive Diagnosis and Treatment of Cancer", Tokushima, Japan \\ IUniversité du Littoral Côte d'Opale, Laboratoire de Physico-Chimie de l'Atmosphère, Dunkerque, France \\ hBeihang University, Beijing Advanced Innovation Center for Big Data-based Precision Medicine, Beijing, China
}

\begin{abstract}
Dual-comb spectroscopy (DCS) is an emerging spectroscopic tool with the potential to simultaneously achieve a broad spectral coverage and ultrahigh spectral resolution with rapid data acquisition. However, the need for two independently stabilized ultrafast lasers significantly hampers the potential application of DCS. We demonstrate mode-resolved DCS in the THz region based on a free-running singlecavity dual-comb fiber laser with the adaptive sampling method. While the use of a free-running single-cavity dual-comb fiber laser eliminates the need for two mode-locked lasers and their frequency control, the adaptive sampling method strongly prevents the degradation of spectroscopic performance caused by the residual timing jitter in the free-running dual-comb laser. Doppler-limit-approaching absorption features with linewidths down to $25 \mathrm{MHz}$ are investigated for low-pressure acetonitrile/air mixed gas by comb-mode-resolved $\mathrm{THz}$ spectroscopy. The successful demonstration clearly indicates its great potential for the realization of lowcomplexity, Doppler-limited THz spectroscopy instrumentation.
\end{abstract}

Keywords: optical comb; terahertz; dual-comb spectroscopy; molecular spectroscopy.

Received Jan. 6, 2020; revised manuscript received Mar. 29, 2020; accepted for publication Apr. 23, 2020; published online May 27, 2020.

(C) The Authors. Published by SPIE and CLP under a Creative Commons Attribution 4.0 Unported License. Distribution or reproduction of this work in whole or in part requires full attribution of the original publication, including its DOI.

[DOI: 10.1117/1.AP.2.3.036004]

\section{Introduction}

Coherent spectroscopic techniques in the terahertz (THz) or farinfrared region (frequencies of 0.1 to $10 \mathrm{THz}$ and wavelengths of $30 \mu \mathrm{m}$ to $3 \mathrm{~mm}$ ) are the enabling technology for a wide variety of important applications. ${ }^{1-3}$ Among these techniques, photonic-based $\mathrm{THz}$ time-domain spectroscopy (THz-TDS ${ }^{4,5}$ has been applied to the study of a diverse range of samples, such as the rotational transitions of polar gas molecules ${ }^{6}$ the hydrogen bonding signature in aqueous systems, ${ }^{7}$ and self-assembly

*Address all correspondence to Zheng Zheng, E-mail: zhengzheng@ buaa.edu.cn; Takeshi Yasui, E-mail: yasui.takeshi@tokushima-u.ac.jp of proteins, ${ }^{8}$ because it takes advantage of the broad spectral bandwidth of THz emitters/receivers pumped by ultrafast lasers. This well-established THz-TDS scheme uses pump pulses and delayed probe pulses from a femtosecond mode-locked laser for $\mathrm{THz}$ radiation and $\mathrm{THz}$ detection, respectively. However, it has limited spectral resolution and accuracy due to constraints on the travel range, repeatability, and speed of the mechanical delay lines. Thus, the use of the photonic $\mathrm{THz}$ techniques for Doppler-limited spectral measurements and quantitative analysis of molecular rotational transitions, which are attractive for many applications including gas sensing, has not yet been well explored. 
Because optical frequency combs $^{9,10}$ provide unprecedented freedom and accuracy in manipulating optical frequencies, dualcomb spectroscopy (DCS $)^{9,10}$ is a powerful tool for overcoming this limitation. The use of two optical combs with a small frequency spacing offset enables us to achieve greatly enhanced spectroscopic performance by avoiding the physical delay line. In the $\mathrm{THz}$ region, $\mathrm{THz}$ dual-comb spectroscopy $(\mathrm{THz}-\mathrm{DCS})^{11-13}$ has become a promising pathway toward ultrahigh-resolution broadband $\mathrm{THz}$ spectroscopy. While two $\mathrm{THz}$ combs can be directly generated from a pair of $\mathrm{THz}$ quantum cascade lasers, ${ }^{14-16}$ such compact $\mathrm{THz}$ sources have a relatively large repetition rate (frequency spacing) between comb modes and often poor mutual coherence between them, leading to coarse sampling spacing and limited spectral resolution. By contrast, if two stabilized optical combs with a highly mutual stability are used for the generation and detection of the $\mathrm{THz}$ comb, the mixing of dual $\mathrm{THz}$ combs with highly mutual coherence can be mapped to an RF comb with the temporal magnification factor (TMF), which is given by the ratio of the repetition rate $f_{\text {rep } 1}$ to the repetition rate offset $\Delta f_{\text {rep }}\left(f_{\text {rep } 1} / \Delta f_{\text {rep }}\right)$. An RF comb is easily accessible with low-bandwidth electronics and further processed to yield high-bandwidth, high-resolution $\mathrm{THz}$ spectroscopic information. THz-DCS enables us to achieve the spectral resolution equal to the $\mathrm{THz}$ comb-mode linewidth, which is on the order of $\mathrm{MHz}$ or better. ${ }^{17,18}$ However, the practical use of mode-resolved THz-DCS is still hampered by the need for two repetitionrate-stabilized optical frequency comb sources because of their expensiveness and complexity in the same manner as optical DCS.

Various schemes have been investigated to further reduce the complexity of THz-DCS and optical DCS systems. Recently, advances in the endeavor to generate a pair of frequency combs from a free-running single-cavity laser ${ }^{19-28}$ have shown great potential toward this goal. By propagating through the same laser cavity, the dual-optical combs experience almost the same disturbances, and the common-mode fluctuations thereby prevent the decline of the mutual coherence between the dual combs. Such a single-cavity dual-comb laser (SCDCL) has been effectively applied to optical DCS ${ }^{25,29-33}$ and THz-DCS ${ }^{34,35}$ with low complexity. The mode-resolved comb spectrum was obtained in optical and THz regions using SCDCL. However, their applications have been limited to pressure-broadening gas spectroscopy with absorption features of sub-GHz to $\mathrm{GHz}$ linewidth ${ }^{31,33}$ due to the limited spectral resolution resulting from the instability of the TMF caused by the residual timing jitter of the SCDCL. Recently, a computational phase correction method has been applied to the optical DCS. Depending on the noise characteristics and available signals, it could compensate for such timing jitters through a computational process after acquiring the interferogram in the time domain. A combination of frequency-stable combs and slow computation correction facilitated the realization of $1-\mathrm{Hz}$ linewidth in the near-infrared region. ${ }^{36}$ The computational approach was also extended to the $\mathrm{THz}$ spectroscopy regime based on quantum cascaded lasers. ${ }^{37}$ It had been applied to the SCDCL for Doppler-limited gas spectroscopy in the nearinfrared region, ${ }^{38}$ with a spectral linewidth of the observed absorption features around $600 \mathrm{MHz}$ at a relatively high $f_{\text {rep1 }}$ $(\sim 142 \mathrm{MHz})$. Adaptive sampling is another attractive technique. By correcting the nonlinearity of the time scale caused by the TMF instability with the adaptive timing clock, the relative coherence between the two combs can be actively recovered for relatively long-time scales without any computational processing of interferograms. It has been demonstrated that a combination of the adaptive sampling method with two freerunning lasers in DCS is more powerful than a combination of a constant sampling method with two stabilized lasers under no further postcorrection. ${ }^{39}$ Alternatively, the digital-domain adaptive sampling approach where the DCS signal is digitized but then resampled could be very effective as well and could deal with even faster noise. ${ }^{24,40-44}$ These adaptive sampling approaches impose mutual coherence between the combs and therefore provide improved spectral resolution. A combination of such adaptive sampling methods with a lower- $f_{\text {rep1 }}$ SCDCL will be the ultimate form of DCS for high spectroscopic performance and reduced system complexity.

In this paper, we demonstrate an adaptive-sampling, nearDoppler-limited THz-DCS scheme based on a simple freerunning SCDCL. Doppler-limit-approaching absorption features were investigated for low-pressure gas mixtures of acetonitrile and air.

\section{Materials and Methods}

\subsection{Principle of Operation}

THz-DCS can be performed in the frequency domain ${ }^{11,12}$ or time domain. ${ }^{13}$ In the time-domain THz-DCS, the $\mathrm{THz}$ frequency comb spectrum is obtained by a combination of asynchronous optical sampling (ASOPS) ${ }^{45-47}$ and Fourier transform (FT), as shown in Fig. 1(a). Using two mode-locked lasers with slightly mismatched repetition rates $\left(f_{\text {rep1 }}\right.$ and $f_{\text {rep2 }}, \Delta f_{\text {rep }}=$ $\left.f_{\text {rep2 }}-f_{\text {rep1 }}\right)$ for $\mathrm{THz}$ generation and detection, a THz pulse train (with a repetition rate of $f_{\text {rep1 }}$, consecutive $\mathrm{THz}$ pulses of $N$, and a time window size of $N / f_{\text {rep1 }}$ ) is slowed down to an RF pulse train (with a repetition rate of $\Delta f_{\text {rep }}$, consecutive RF pulses of $N$, and a time window size of $N / \Delta f_{\text {rep }}$ ) by the TMF $\left(f_{\text {rep1 }} / \Delta f_{\text {rep }}\right)$ based on the temporal magnifying function of ASOPS; this enables a direct acquisition of the RF pulse train using a data acquisition board without the need for mechanical time-delay scanning. FT of the RF pulse train results in the mode-resolved RF comb spectrum with a frequency spacing of $\Delta f_{\text {rep }}$. Finally, the mode-resolved THz comb spectrum is obtained by calibrating the frequency scale of the RF comb spectrum with the inverse of the TMF. The mode-resolved $\mathrm{THz}$ comb spectrum contains a series of successive narrow lines with a frequency spacing equal to the repetition rate $\left(f_{\text {rep1 }}\right)$ and a spectral sampling step equal to the inverse of the time window $\left(f_{\text {rep1 }} / N\right)$. The linewidth of each comb mode is equal to the spectral sampling step.

Details of the adaptive sampling method have been discussed elsewhere, ${ }^{39}$ so only its brief description is given here. If the TMF is fluctuated by the residual timing jitter of the free-running SCDCL, linearity of the time scale of the RF pulse train is distorted as shown in the upper row of Fig. 1(b). Such temporal nonlinearity is transferred to the frequency scale of the RF comb and $\mathrm{THz}$ comb, which will seriously degrade the spectral resolution. If the RF pulse train is acquired by a sampling clock synchronized with the residual timing jitter, namely an adaptive clock, as shown in the middle row of Fig. 1(b), the time-scale linearity of the sampled signal can be recovered [see the lower row of Fig. 1(b)]. In this case, the time window size can be effectively extended without an accumulation of timing errors. It is also possible to acquire accumulated data over a longer period of time to realize an improved SNR. 


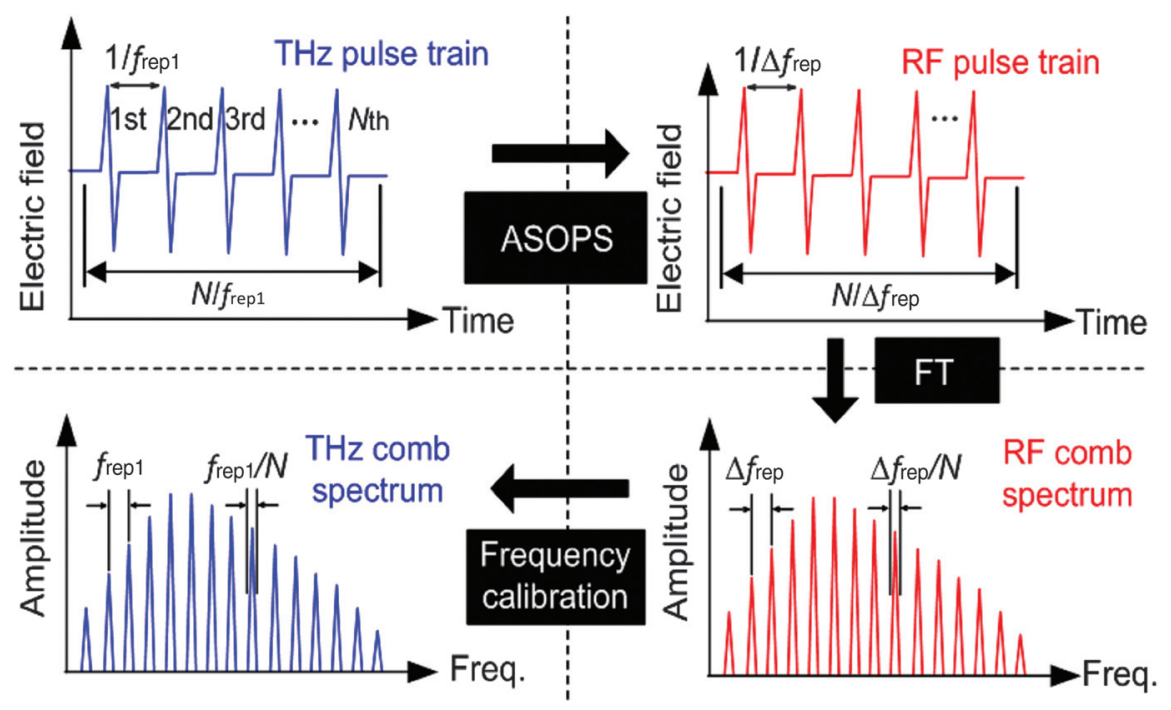

(a)

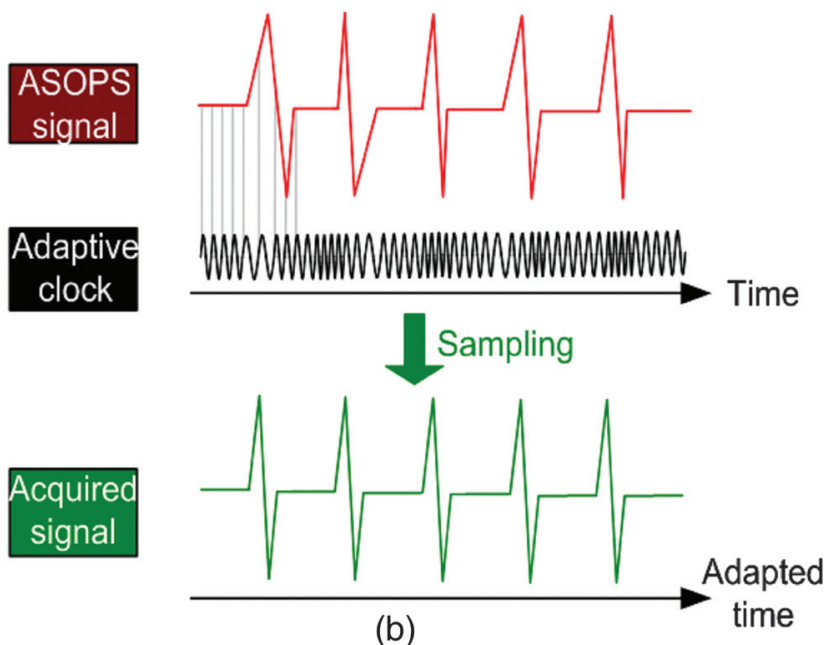

(b)

Fig. 1 Principle of operation. (a) Flowchart of time-domain THz-DCS. (b) Acquisition of the temporal waveform using the adaptive sampling method.

\subsection{Single-Cavity Dual-Comb Laser}

The upper-left part of Fig. 2 shows a free-running SCDCL composed of an all-fiber ring-cavity oscillator, a band-pass filter, and erbium-doped fiber amplifiers (EDFAs). The dual-comb light beams with different center wavelengths and different $f_{\text {rep }}$ were obtained from the fiber oscillator by multiplexing the modelocking operation in the wavelength region. ${ }^{29,32,34}$ The fiber oscillator has an all-fiber ring cavity in which the dual-comb light beams propagate along a common-path route. The cavity consists of a hybrid wavelength division multiplexer and isolator (WDM/ISO), a piece of 0.46-m-long erbium-doped fiber (EDF) pumped by a $980-\mathrm{nm}$ laser diode, a polarization controller (PC), a single-wall carbon nanotube saturable absorber (SA), an inline polarizer (ILP) with polarization-maintained fiber (PMF) pigtails, and a $40 \%$ fiber output coupler (OC). In addition, a 65-cm-long dispersion compensation fiber was installed to optimize the intracavity dispersion at $\sim 4.0 \mathrm{fs} / \mathrm{nm}$ at $1560 \mathrm{~nm}$. The total length of the cavity was $\sim 4.21 \mathrm{~m}$. Due to the considerable birefringence of the PMF and the use of the ILP, spectral filtering and polarization-dependent loss tuning enable dual-comb lasing oscillation by adjusting the PC. ${ }^{29}$ Dual-comb light with different center wavelengths and different $f_{\text {rep }}\left(\lambda_{1}\right.$-comb with $f_{\text {rep } 1}$ and $\lambda_{2}$-comb with $f_{\text {rep2 } 2}$ ) from the oscillator was separated into two independent combs with different center wavelengths and different $f_{\text {rep }}$ by the bandpass filter, and the resulting two combs were further amplified by EDFAs. The difference in their center wavelengths is negligible for generation and detection of $\mathrm{THz}$ comb.

\subsection{Adaptive Clock Generator}

The adaptive clock generator (ACG) provides the adaptive clock to suppress the residual long-term drift and timing jitter in the repetition rate difference $\Delta f_{\text {rep }}$ of the free-running SCDCL, enabling us to recover the temporal linearity of the acquired temporal waveform. To trace the timing fluctuation in real time, we used photoconductive heterodyning mixing between a reference $\mathrm{CW}-\mathrm{THz}$ radiation and two photocarrier $\mathrm{THz}$ combs $(\mathrm{PC}-\mathrm{THz}$ comb1 with $f_{\text {rep } 1}$ and PC-THz comb2 with $f_{\text {rep2 }}$ ) seeded by the SCDCL output, ${ }^{39}$ as shown in the middle part of Fig. 2. Two bowtie-shaped, low-temperature-grown GaAs photoconductive 


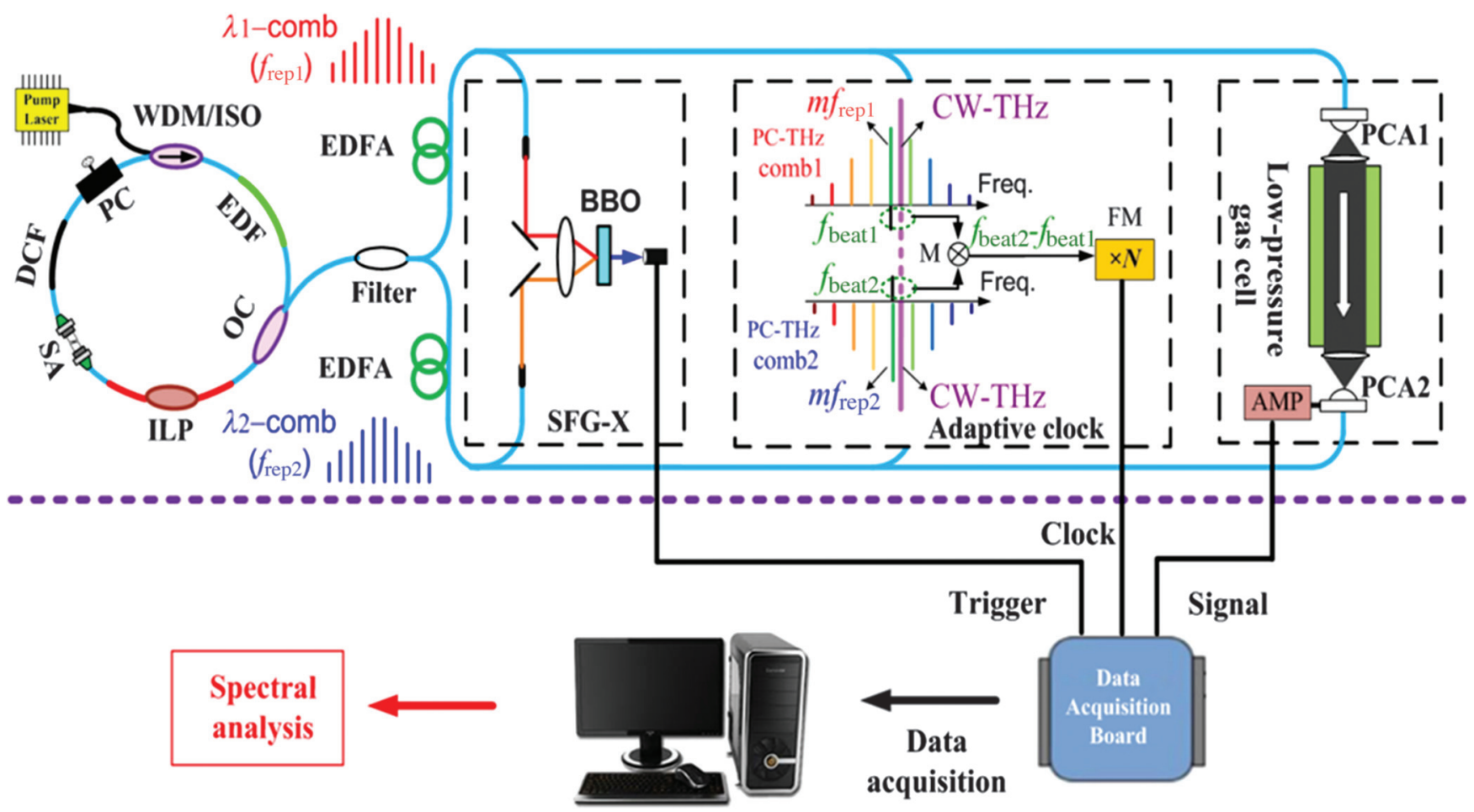

Fig. 2 Configuration of comb-mode-resolved adaptive sampling THz-DCS. SFG-X, sum-frequencygeneration cross-correlator; $\mathrm{BBO}$, beta-barium borate crystal; $\mathrm{PC}-\mathrm{THz}$ comb, photocarrier $\mathrm{THz}$ comb; M, double-balanced mixer; FM, frequency multiplier (frequency multiplication factor $N=40$ ); PCA, photoconductive antenna; and AMP, current preamplifier.

antennas (BT-PCA1 and BT-PCA2) were optically excited by the second-harmonic-generation light of the optical dual combs (not shown in Fig. 2). When the $\mathrm{CW}-\mathrm{THz}$ radiation $\left(f_{\mathrm{THz}}=\right.$ $0.1 \mathrm{THz}$, linewidth $<0.6 \mathrm{~Hz}$, and average power $=2.5 \mathrm{~mW}$ ) from an active frequency multiplier (FM) chain sourced by a microwave frequency synthesizer was also incident on both BT-PCA1 and BT-PCA2, two RF beat signals were generated between the CW-THz and the nearest adjacent PC-THz comb modes. Their frequencies $\left(f_{\text {beat } 1}\right.$ and $\left.f_{\text {beat } 2}\right)$ are given by $\mid f_{\mathrm{THz}}-$ $m f_{\text {rep } 1} \mid$ and $\left|f_{\mathrm{THz}}-m f_{\text {rep2 } 2}\right|$, respectively, assuming that the same $m$-order comb lines are involved. The $f_{\text {beat } 2}-f_{\text {beat } 1}$ signal that carries the timing fluctuation information $\left(=m f_{\text {rep } 2}-\right.$ $m f_{\text {rep1 }}=m \Delta f_{\text {rep }}$ ) was obtained by electrical mixing in a double-balanced mixer $(\mathrm{M})$. The resulting signal was further frequency-multiplied by an FM (frequency multiplication factor $N=40$ ), which serves as the adaptive sampling clock.

\subsection{THz Dual-Comb Spectroscopy}

The right part of Fig. 2 shows the experimental setup of THzDCS. THz comb was radiated from a fiber-coupled, strip-lineshaped LT-InGaAs/InAlAs photoconductive antenna (PCA1, TERA 15-TX-FC, Menlo Systems, bias voltage $=20 \mathrm{~V}$, optical power of $20 \mathrm{~mW}$ ) excited by $\lambda_{1}$-comb pump light and then passed through a low-pressure gas cell (length $=38 \mathrm{~cm}$, diameter $=17 \mathrm{~mm}$ ). The $\mathrm{THz}$ comb was then detected by another fiber-coupled, dipole-shaped LT-InGaAs/InAlAs photoconductive antenna (PCA2, TERA 15-RX-FC, Menlo Systems, optical power $=20 \mathrm{~mW}$ ) that was pumped by the $\lambda_{2}$-comb light.
The electrical output of PCA2 was amplified by a current preamplifier (AMP, bandwidth $=3.8 \mathrm{MHz}$, gain $=1 \times 10^{6} \mathrm{~V} / \mathrm{A}$ ).

A portion of the separated $\lambda_{1}$-comb and $\lambda_{2}$-comb lights was fed into a sum-frequency-generation cross-correlator (SFG-X), with a setup that was constructed based on a noncollinear configuration with a piece of $\beta-\mathrm{BaB}_{2} \mathrm{O}_{4}$ (BBO) crystal. The resulting SFG pulse that occurs every $1 / \Delta f_{\text {rep }}$ served as the trigger signal for the data acquisition board.

The temporal waveform of the amplifier output was acquired with a data acquisition board. The mode-resolved $\mathrm{THz}$ comb spectrum was obtained by taking Fourier transform of the temporal waveform accumulated in the time domain. A rubidium frequency standard (Stanford Research FS725, accuracy $=5 \times$ $10^{-11}$ and instability $=2 \times 10^{-11}$ at $1 \mathrm{~s}$ ) is used to provide the common time-base signal for the CW-THz source and the data acquisition board (not shown in Fig. 2).

\subsection{Sample}

Acetonitrile $\left(\mathrm{CH}_{3} \mathrm{CN}\right)$ is a symmetric top molecule with a rotational constant $B$ of $9.194 \mathrm{GHz}$ and a centrifugal distortion constant $D_{J K}$ of $17.74 \mathrm{MHz},{ }^{48}$ and its gas phase has relatively complicated spectral absorption features in the $\mathrm{THz}$ domain. Frequencies of its rotational transitions are given as

$v=2 B(J+1)-2 D_{J K} K^{2}(J+1)$,

where $J$ and $K$ are rotational quantum numbers. There are two sets of characteristic features in the $\mathrm{THz}$ spectrum of $\mathrm{CH}_{3} \mathrm{CN}$. 
From the first term in Eq. (1), there are multiple manifolds of rotational transitions that are equally spaced by $2 B$ $(\approx 18.388 \mathrm{GHz})$. From the second term in Eq. (1), each manifold further consists of a series of closely spaced absorption lines on the order of tens of MHz. The MHz-order absorption feature makes this gas a good candidate for the demonstration of Doppler-limited gas spectroscopy in the $\mathrm{THz}$ region.

\section{Results}

\subsection{Basic Performance of SCDCL}

When the cavity EDF was pumped beyond its mode-locking threshold and the intracavity PC was properly adjusted, dualcomb mode-locking oscillation was achieved at two different center wavelengths with similar peak intensities, as shown in Fig. 3(a). Two spectral peaks at 1532.5 and $1557.7 \mathrm{~nm}$ have $3 \mathrm{~dB}$ bandwidths of 4.2 and $3.6 \mathrm{~nm}$, respectively, corresponding to the $\lambda_{1}$-comb light and $\lambda_{2}$-comb light. Due to the finely adjusted, low intracavity anomalous dispersion, the $\lambda_{1}$-comb light and $\lambda_{2}$-comb light have slightly different repetition rates, $f_{\text {rep1 }}$ $(\sim 48.804486 \mathrm{MHz})$ and $f_{\text {rep2 }}(\sim 48.804296 \mathrm{MHz})$, and their difference $\left(\Delta f_{\text {rep }}\right)$ is only $\sim 190 \mathrm{~Hz}$, as shown in Fig. 3(b). After separate amplification by EDFAs, the dual-comb lights are spectrally broadened and temporally compressed to $\sim 110 \mathrm{fs}$ at full-width-at-half-maximum (FWHM) by propagating through the standard single-mode fiber. The achieved pulse duration is sufficient to drive the broadband $\mathrm{THz}$ comb spectrum.

The temporal drifts of $f_{\text {rep } 1}, f_{\text {rep } 2}$, and $\Delta f_{\text {rep }}$ under freerunning conditions were monitored in time because they alter the TMF between the THz comb and RF comb. As shown in Fig. 3(c), the drifts of both repetition rates are $\sim 2.5 \mathrm{~Hz}$ in $120 \mathrm{~s}$ and follow the same trend owing to the identical fluctuations in the completely shared path for both pulses. The resulting fluctuation of $\Delta f_{\text {rep }}$ is merely $5.1 \mathrm{mHz}$ in terms of standard deviation without active stabilization. The frequency instability of $f_{\text {rep } 1}$ and $\Delta f_{\text {rep }}$ in the SCDCL was measured for different gate times as indicated by the red solid and hollow circles in Fig. 3(d). For comparison, we also evaluated the frequency instability of $f_{\text {rep1 }}$ and $\Delta f_{\text {rep }}$ for two independently stabilized fiber-comb lasers [see the blue solid and hollow circles in Fig. 3(d)] and two independently free-running fiber-comb lasers [see the green solid and hollow circles in Fig. 3(d)]. When comparing the SCDCL with two independently free-running fiber-comb lasers, $f_{\text {rep } 1}$ and $\Delta f_{\text {rep }}$ show similar trends. The fluctuation of $f_{\text {rep } 1}$ for the SCDCL is nearly one order larger than that of free-running lasers when the gate time is more than $1 \mathrm{~s}$, which might be due to the better outer packaging of the fibercomb laser for environmental immunity. However, the frequency stability of $\Delta f_{\text {rep }}$ in the SCDCL was significantly better than that in the two free-running fiber-comb lasers over a short time. Next, when comparing the SCDCL with two independently stabilized fiber-comb lasers, the SCDCL shows better short-term stability in $\Delta f_{\text {rep }}$ than the two independently stabilized fiber-comb lasers. By contrast, the two independently stabilized fiber-comb lasers show better long-term stability in $\Delta f_{\text {rep }}$ and $f_{\text {rep } 1}$ than the SCDCL. Importantly, even though the SCDCL is inferior to the two independently stabilized fiber-comb lasers in terms of the long-term stability of $\Delta f_{\text {rep }}$ and $f_{\text {rep } 1}$ due to the lack of active laser stabilization, the adaptive sampling method allows us to overcome such inferiority significantly as demonstrated later, leading to a reduced system complexity and excellent spectroscopic performance.

\subsection{Performance of Adaptive Sampling THz-DCS}

To investigate the effectiveness of the proposed adaptive sampling THz-DCS method, 100,000 temporal waveforms of
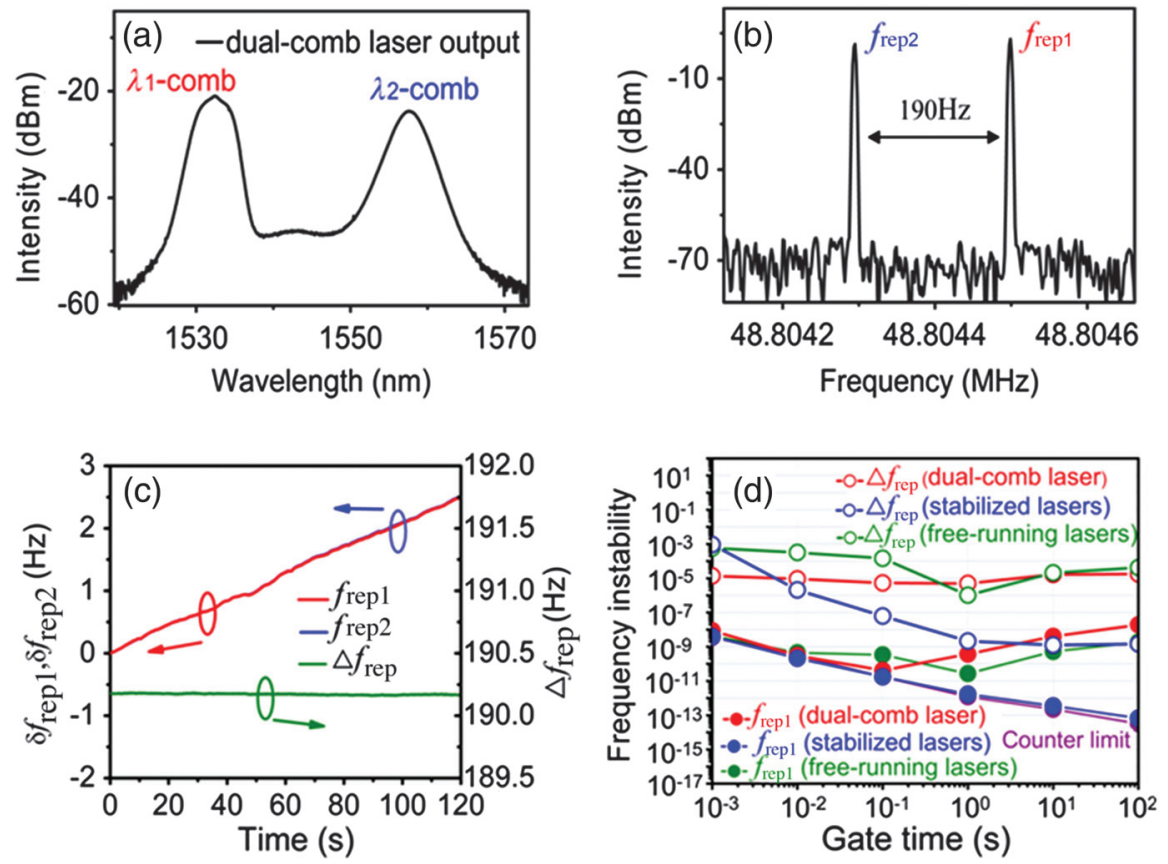

Fig. 3 Performance of the dual-comb fiber laser. (a) Output spectrum of the laser. (b) RF spectrum of the dual-comb pulses. (c) Fluctuations of $f_{\text {rep1 } 1}, f_{\text {rep2 }}$, and $\Delta f_{\text {rep }}$ (d) Measured frequency instability of $f_{\text {rep } 1}$ and $\Delta f_{\text {rep. }}$. 
10-consecutive $\mathrm{THz}$ pulse train were acquired and accumulated by the adaptive sampling method. For a comparison, similar temporal waveforms were acquired based on a constant sampling method, which is widely used for data acquisition of the previous DCS with SCDCL. ${ }^{31,33}$ In the case of the constant sampling method as shown in the upper part of Fig. 4(a), the $\mathrm{THz}$ pulses almost disappeared except for the first pulse because the residual timing jitter causes random walk-off of the temporal sampling positions in each time-delay scan, leading to a low efficiency in the signal accumulation. Obviously, when the number of signal accumulation is largely increased, the constant sampling method with the SCDCL is not suitable to extend the temporal window of the accumulated temporal waveform up to multiple pulse periods. Therefore, previous DCS with SCDCL was demonstrated in a short acquisition time of the temporal waveform (typically, $<1 \mathrm{~s}$ ). ${ }^{31,33}$ When the adaptive sampling method was used for THz-DCS with SCDCL, each $\mathrm{THz}$ pulse was clearly observed in the accumulated temporal waveform, as shown in the lower part of Fig. 4(a). Moreover, each $\mathrm{THz}$ pulse was the complete duplicate of each other, coincident with the behavior of $\mathrm{THz}$ comb in the time domain. As shown in the inset of Fig. 4(a), the asymmetric bipolar monocycle pulse shape is due to the dipole radiation from the photoconductive antenna, with its temporal waveform given by a time derivative of transient photocurrent induced by the pump light.
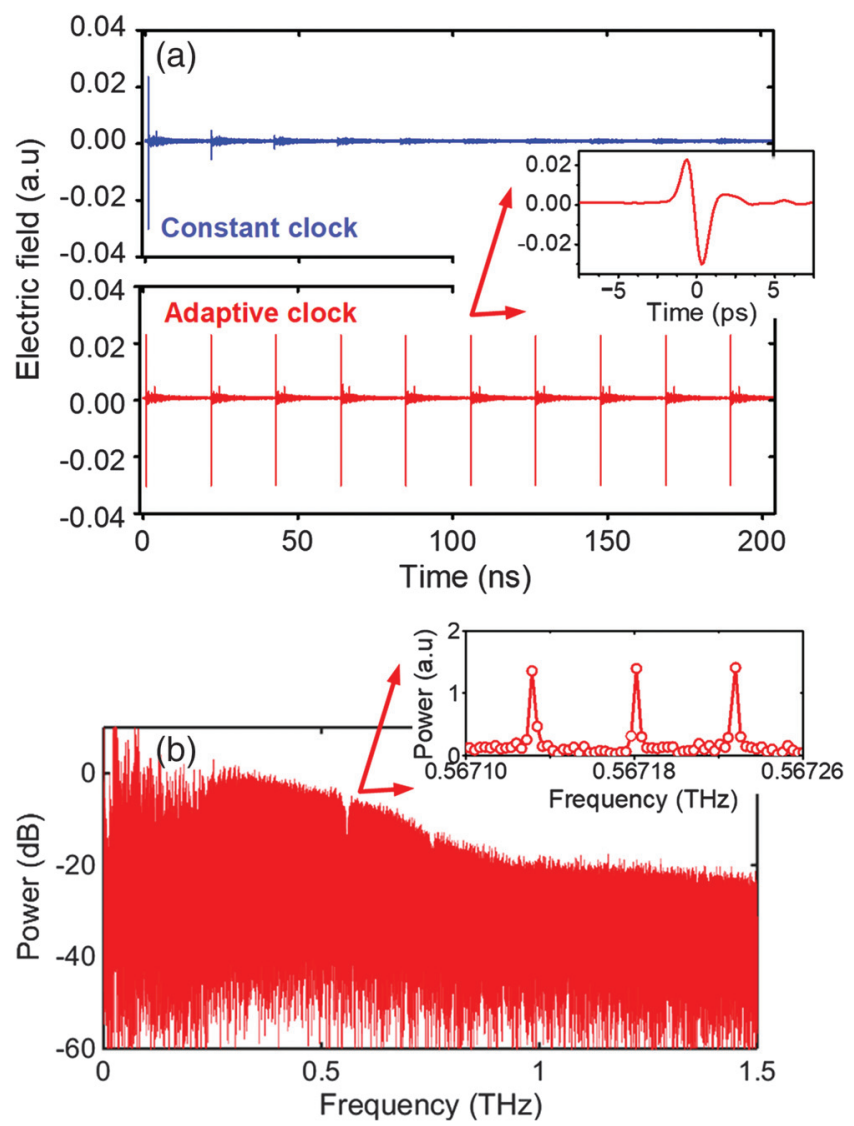

Fig. 4 (a) Comparison of the temporal waveforms averaged 100,000 times obtained using different sampling clocks. Inset: a zoomed-in plot of the main $\mathrm{THz}$ pulse. (b) Comb-moderesolved $\mathrm{THz}$ spectrum through air at room pressure. Inset: a zoomed-in plot around $0.5672 \mathrm{THz}$.
These results imply that the adaptive sampling method has the capability to significantly suppress the instability of TMF over a long data acquisition time.

Next, the mode-resolved $\mathrm{THz}$ comb spectrum was obtained by calculating the FT of the temporal waveform of the adaptive sampling RF pulse train [see the lower part of Fig. 4(a)], as shown in Fig. 4(b). A $\sim 50-\mathrm{dB}$ power dynamic range was achieved in the $0.1-$ to $1.5-\mathrm{THz}$ frequency band. An expanded view of the spectral region around $0.567 \mathrm{THz}$ is shown in the inset of Fig. 4(b), indicating multiple discrete lines with a constant frequency spacing of $48.8 \mathrm{MHz}$ exactly equal to $f_{\text {rep } 1}$. The spectral width of each comb line was $4.88 \mathrm{MHz}$, which is exactly equal to the theoretical FT spectral resolution given by an inverse of the time window size ( $f_{\text {repl }} / 10$ in this case). Because we later investigate the pressure-broadening characteristics of the sample gas within the range of absorption linewidth from a few tens $\mathrm{MHz}$ to $100 \mathrm{MHz}$, the comb-mode linewidth was set to be $f_{\text {rep } 1} / 10(=4.88 \mathrm{MHz})$ to acquire the spectrum with a minimum required resolution and data size. In the previous study, the constant sampling THz-DCS with dual stabilized lasers could be demonstrated with the time window size of $100 / f_{\text {rep } 1},{ }^{49,50}$ whereas the adaptive sampling THz-DCS with dual free-running lasers was more powerful than the constant sampling THz-DCS with dual stabilized lasers. ${ }^{39}$ We consider that there is a space to further decrease the comb-mode linewidth in the present system. In this way, the combination of the adaptive sampling method with the SCDCL has the potential to reach spectral resolution of $\mathrm{MHz}$ level or below without the influence of the residual timing jitter in SCDCL. In Fig. 4(b), two spectral dips appeared at 0.557 and $0.752 \mathrm{THz}$ with an FWHM linewidth of $7 \mathrm{GHz}$ in the mode-resolved $\mathrm{THz}$ comb spectrum, which was caused by atmospheric water vapor existing in the $\mathrm{THz}$ optical path outside the gas cell. This is a simple demonstration of pressure-broadening gas spectroscopy in the $\mathrm{THz}$ region.

\subsection{Near-Doppler-Limited Spectroscopy of Low-Pressure Acetonitrile/Air Gas}

To demonstrate the high-resolution spectroscopic capability of the proposed system, $\mathrm{THz}$ spectroscopy of mixed gas of acetonitrile $\left(\mathrm{CH}_{3} \mathrm{CN}\right)$ and air was performed at low pressures. $\mathrm{CH}_{3} \mathrm{CN}$ has a rotational constant $B$ of $9.194 \mathrm{GHz}$ and a centrifugal distortion constant $D_{J K}$ of $17.74 \mathrm{MHz}^{48}$ and is a good candidate for the demonstration of Doppler-limited gas spectroscopy in the $\mathrm{THz}$ region because of the closely spaced rotational transitions on the order of tens of $\mathrm{MHz}$ in each manifold. The gas cell was filled with a mixture of $\mathrm{CH}_{3} \mathrm{CN}$ and air with a total pressure of $360 \mathrm{~Pa}$. The absorption spectrum of this mixed gas was obtained by normalizing the mode-resolved $\mathrm{THz}$ comb spectrum measured in the $\mathrm{CH}_{3} \mathrm{CN} /$ air-filled gas cell with that measured in the vacuum gas cell. Figure 5(a) shows the broadband spectrum of absorbance coefficient within the frequency range of 0.2 to $0.7 \mathrm{THz}$. 29 manifolds were observed with a frequency separation of $18.388 \mathrm{GHz}$ equal to $2 B$ and were correctly assigned to $J=10$ to 38 by comparison with the Jet Propulsion Laboratory (JPL) spectral database. ${ }^{51}$ Figure 5(b) shows a zoomed-in plot of Fig. 5(a) within the frequency range of 0.31 to $0.37 \mathrm{THz}$. In addition to $J=16$ to 19 at the ground state, the manifolds of the rotational transitions at the vibrationally excited states ${ }^{49}$ were clearly observed as marked by the red asterisks. These spectral features could be obtained by 

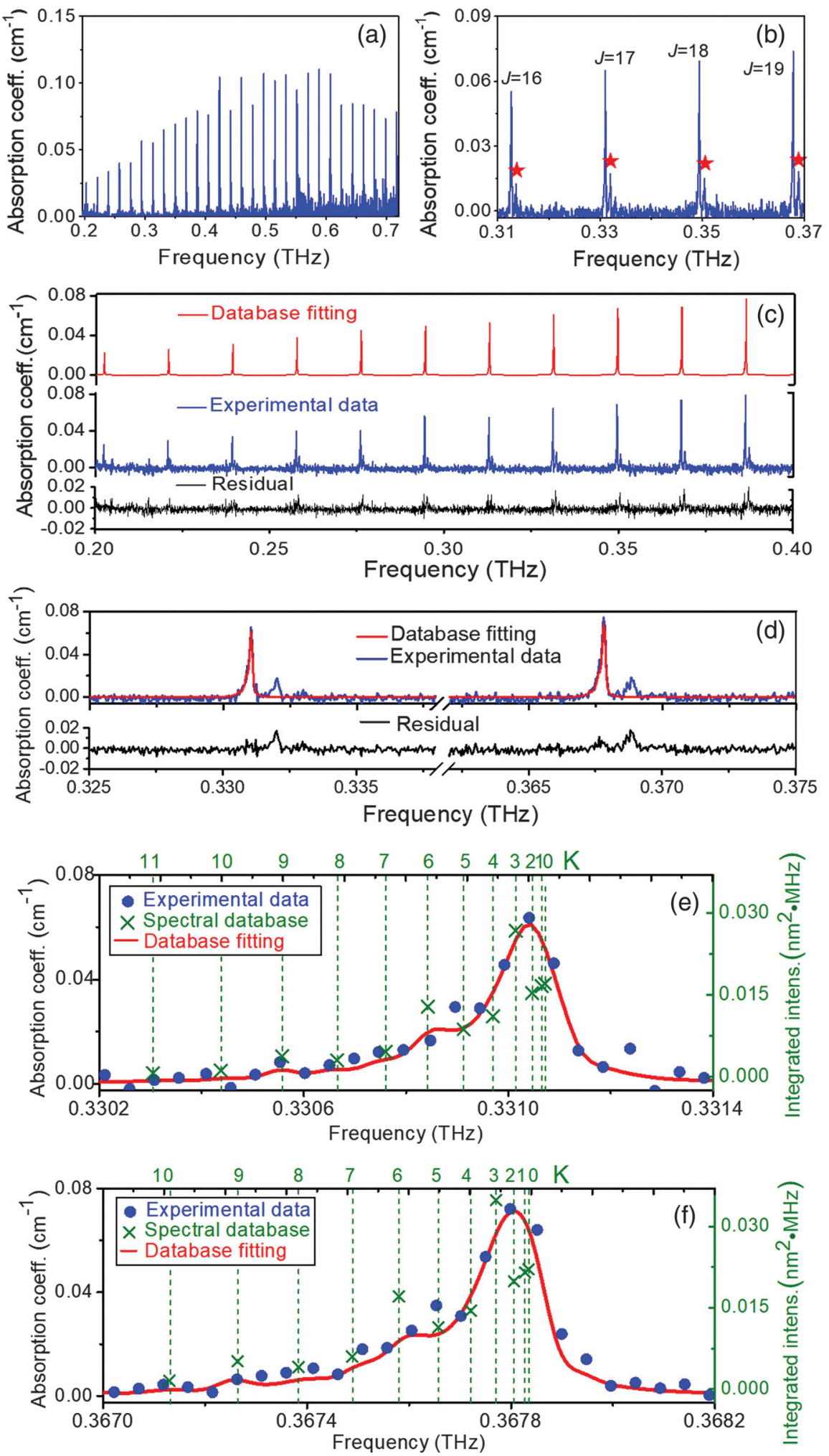

Fig. 5 Comb-mode-resolved THz spectroscopy of a mixture gas sample of $\mathrm{CH}_{3} \mathrm{CN}$ and air with a total pressure of $360 \mathrm{~Pa}$. Absorption spectra of $\mathrm{CH}_{3} \mathrm{CN}$ within the frequency range of (a) 0.2 to $0.72 \mathrm{THz}$ and (b) 0.31 to $0.37 \mathrm{THz}$. The red stars indicate the manifolds of the rotational transitions for the vibrationally excited states. (c) Comparison of the absorption spectra between the database fitting and the experimental data and their residual and (d) the corresponding zoomedin plot around 0.3310 and $0.3677 \mathrm{THz}$. (e) Absorption spectra around $0.3310 \mathrm{THz}$ and (f) $0.3677 \mathrm{THz}$. 
the enhanced spectral resolving power and sensitivity in the lowpressure gas condition, which constitutes a significant enhancement in the spectroscopic performance compared with the previous demonstration. ${ }^{34}$

Each manifold is composed of a number of closely spaced rotational transitions, assigned by $K$, as tabulated in the JPL spectral database. ${ }^{51}$ Multipeak fitting analysis was performed to determine a $\mathrm{CH}_{3} \mathrm{CN}$ partial pressure and the corresponding linewidth of the rotational transitions in which a Lorentzian lineshape was used for each rotational line and a global linewidth parameter was applied to all of the lines. The line positions and intensities were fixed parameters, while the acetonitrile partial pressure and linewidth were left as free parameters. An example is presented in the red and blue plots of Fig. 5(c), yielding a $\mathrm{CH}_{3} \mathrm{CN}$ partial pressure of $127( \pm 1) \mathrm{Pa}$ and an FWHM linewidth of $77( \pm 2) \mathrm{MHz}$. The database fitting curve (red line) was in good agreement with the experimental data (blue line) for all peaks in the frequency range of 0.2 to $0.4 \mathrm{THz}$ with a slight residual (black line). The reason for the periodic modulation of the residual is that the fitting model does not include the rotational transitions at the vibrationally excited states. ${ }^{51}$ This is better illustrated in an expanded view in Fig. 5(d) around 0.3310 and $0.3677 \mathrm{THz}$, which shows that the large residual is mostly around the vibrationally excited states. Figures 5(e) and 5(f) show zoomed-in spectra for $J=17$ at $0.3310 \mathrm{THz}$ and $J=$ 19 at $0.3677 \mathrm{THz}$, in which the blue plots show the experimental data. The frequency spacing of the experimental data points, corresponding to the spectral sampling interval, was $48.8 \mathrm{MHz}$, equal to $f_{\text {rep } 1}$. The curve fitting results based on multipeak fitting analysis are shown by the red line while literature values of JPL spectral database are shown by the green line in Figs. 5(e) and 5(f). Most of the absorption lines have frequency separation between each other equal to or less than the spectral sampling interval (see green lines and blue plots).
Such undersampling acquisition of fine spectral features leads to disagreement between experimental plots and absorption line positions. However, it is important to note that even such an undersampling spectral acquisition is effective for nearDoppler-limited gas spectroscopy with the help of curve fitting analysis, which is discussed later.

The validity of the adaptive sampling THz-DCS scheme was more precisely evaluated by measuring the pressure broadening characteristics of $\mathrm{CH}_{3} \mathrm{CN}$ rotation transition in the $\mathrm{CH}_{3} \mathrm{CN} /$ airmixed gas. Similar to the procedure for $360 \mathrm{~Pa}$ as shown in Fig. 5, a series of absorption spectra of rotational transitions in $J=17$ at $0.331 \mathrm{THz}$ was measured when reducing the total pressure of the $\mathrm{CH}_{3} \mathrm{CN} /$ air-mixed gas from 430, 330, 280, 256, 149 , to $115 \mathrm{~Pa}$ with an uncertainty of $\pm 2 \mathrm{~Pa}$, as shown by blue plots in Figs. 6(a)-6(f), respectively. To perform a quantitative analysis, we again performed multipeak fitting analysis based on a Lorentzian lineshape under the assumption that all $K$ lines have the same linewidth. The total pressure was measured by a pressure gauge to help achieve a sample with the desired approximate concentration and was not used in the fitting procedure. As shown by the red lines in Fig. 6(a)-6(f), the curve fitting spectra match the experimental spectra very well. Both the experimental data and the curve fitting results clearly indicate the pressure-dependent change in the shape of the absorption spectrum. Table 1 shows the result of quantitative analysis for the $\mathrm{CH}_{3} \mathrm{CN}$ partial pressure and the corresponding linewidth of rotational transition with respect to different total pressures of this mixed gas sample. The uncertainties provided in Table 1 are the confidence intervals calculated as part of the fitting procedure. The partial pressure and the linewidth were determined with good uncertainty under all total pressures, indicating the validity of the adaptive sampling THz-DCS and the following curve fitting analysis. In particular, the linewidth was determined to be $25 \mathrm{MHz}$ at a total pressure of $115 \mathrm{~Pa}$. This quantitative
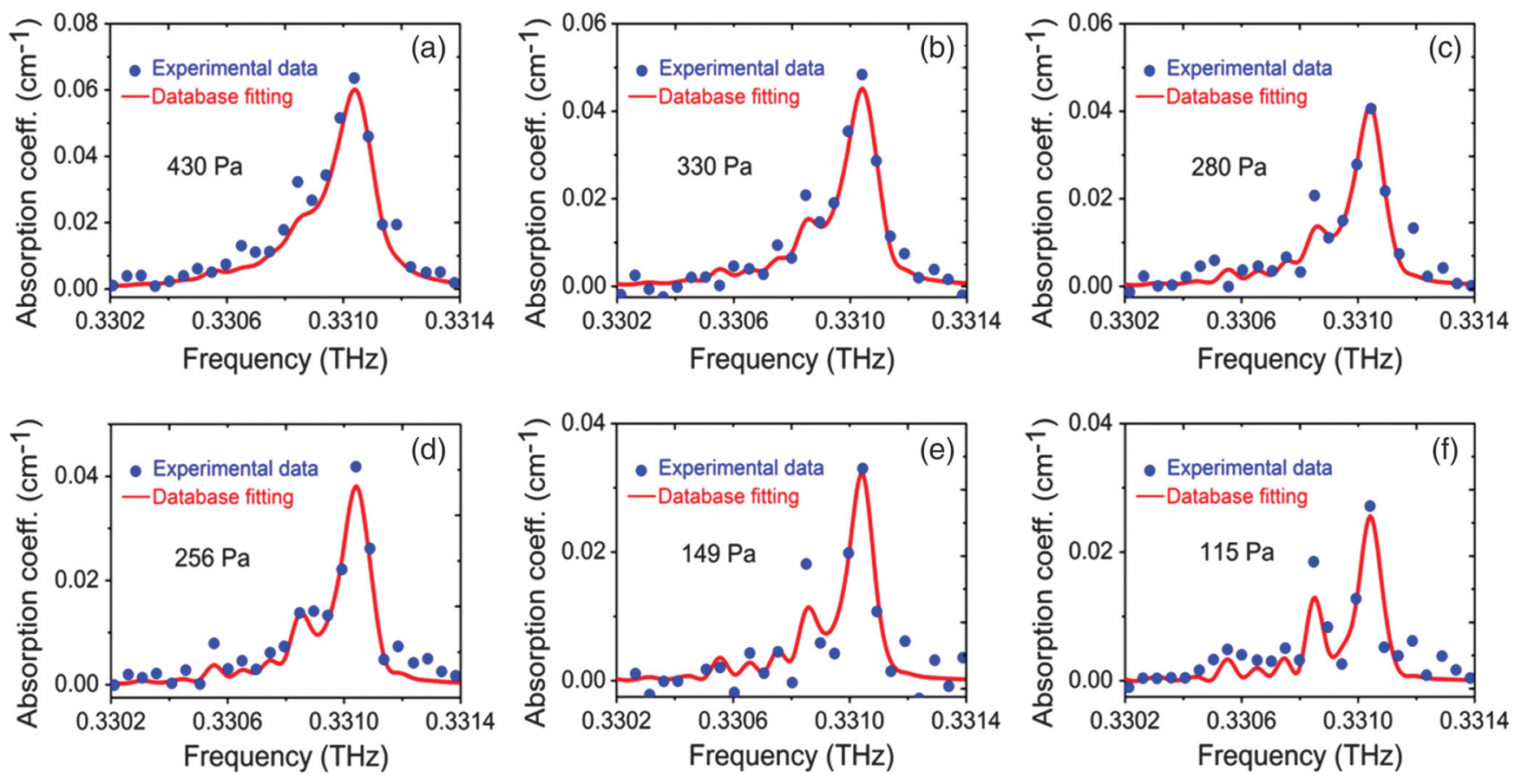

Fig. 6 Mode-resolved absorption characterization of $\mathrm{CH}_{3} \mathrm{CN}$ around $0.331 \mathrm{THz}$ at (a) $430 \mathrm{~Pa}$, (b) $330 \mathrm{~Pa}$, (c) $280 \mathrm{~Pa}$, (d) $256 \mathrm{~Pa}$, (e) $149 \mathrm{~Pa}$, and (f) $115 \mathrm{~Pa}$. 
Table 1 Quantitative analysis of $\mathrm{CH}_{3} \mathrm{CN}$ /air-mixed gas.

\begin{tabular}{|c|c|c|c|c|}
\hline \multirow{2}{*}{$\begin{array}{l}\text { Total pressure of } \\
\mathrm{CH}_{3} \mathrm{CN} / \text { air mixed } \\
\text { gas }(\mathrm{Pa})\end{array}$} & \multicolumn{2}{|c|}{$\begin{array}{c}\text { Partial pressure } \\
\text { of } \mathrm{CH}_{3} \mathrm{CN} \text { gas } \\
(\mathrm{Pa})\end{array}$} & \multicolumn{2}{|c|}{$\begin{array}{c}\text { FWHM linewidth of } \\
\mathrm{CH}_{3} \mathrm{CN} \text { rotation } \\
\text { transition }(\mathrm{MHz})\end{array}$} \\
\hline & Result & Uncertainty & Result & Uncertainty \\
\hline 430 & 142 & 1 & 102 & 2 \\
\hline 360 & 127 & 1 & 77 & 2 \\
\hline 330 & 87 & 1 & 65 & 2 \\
\hline 280 & 73 & 1 & 53 & 2 \\
\hline 256 & 68 & 1 & 47 & 1 \\
\hline 149 & 52 & 1 & 37 & 1 \\
\hline 115 & 42 & 1 & 25 & 1 \\
\hline
\end{tabular}

analysis indicates the capability of the proposed system to realize THz spectroscopy of MHz-order absorption features with the reduced system complexity.

\section{Discussion}

We first discuss the potential of the adaptive sampling THz-DCS scheme for Doppler-limited gas spectroscopy. We confirmed clear differences in the absorption linewidth as the gas pressure was reduced, as shown in Fig. 6 and Table 1. Figure 7 shows a relation between partial pressure of $\mathrm{CH}_{3} \mathrm{CN}$ and absorption linewidth, namely the pressure broadening characteristic, of this mixed gas sample. The linear trend between them indicates that the pressure broadening characteristic of this mixed gas was correctly measured by the proposed system and the following quantitative analysis. We consider that the deviation from a strict linear relationship is caused by other experimental uncertainties such as a drift of TMF because the adaptive sampling corrects the nonlinearity of the time scale but does not ensure the accuracy of the time scale. From the slope of the linear trend, the observed pressure broadening coefficient lies between the values for self-broadening $(912 \mathrm{kHz} / \mathrm{Pa})$ and broadening by nitrogen $(91.2 \mathrm{kHz} / \mathrm{Pa}) .^{52,53}$ The Doppler-limited linewidth of $\mathrm{CH}_{3} \mathrm{CN}$ gas was $382 \mathrm{kHz}$ (FWHM) at $0.2 \mathrm{THz}$ and $1.34 \mathrm{MHz}$ (FWHM) at $0.7 \mathrm{THz}{ }^{54}$ Consistent with these values, the adaptive sampling THz-DCS has a potential to interrogate low-pressure gases with absorption features approaching the Doppler limit.

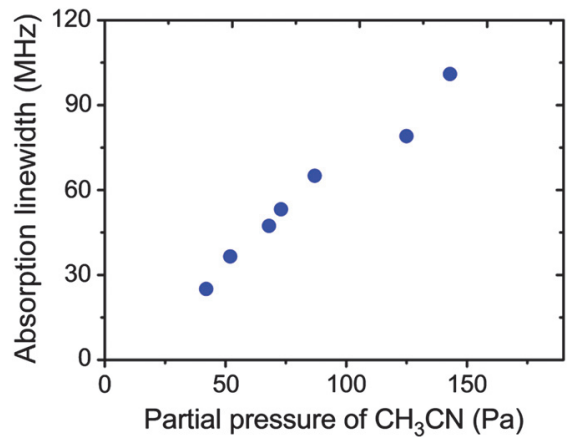

Fig. 7 Pressure broadening characteristic of $\mathrm{CH}_{3} \mathrm{CN}$ /air gas.
We next discuss the possibility of the proposed method for $\mathrm{THz}$ spectroscopy with further enhanced precision. Although we demonstrated the near-Doppler-limited gas spectroscopy in the $\mathrm{THz}$ region, the frequency comb spacing $f_{\text {rep } 1}$ of $48.8 \mathrm{MHz}$ is still large for a full analysis of rotational transitions with $\mathrm{MHz}$-order structure. A promising approach for this purpose is to use the gapless technique in the $\mathrm{THz}$ comb, in which the frequency spacing of two stabilized $\mathrm{THz}$ combs is precisely swept to interleave additional comb lines into the original comb lines. ${ }^{49,50}$ Based on the simple structure of the dual-comb fiber laser, it is also foreseeable to achieve a higher resolution equal to the comb linewidth $(=4.88 \mathrm{MHz})$ by shifting the comb lines through tuning the laser cavity. However, to expand this technique into the free-running $\mathrm{THz}$ combs generated by SCDCL, we have to consider the frequency instability of the $\mathrm{THz}$ comb line because it determines the spectrally interleaved interval. Although the frequency instability of the $\mathrm{THz}$ comb line depends on the fluctuations of both $\Delta f_{\text {rep }}$ and $f_{\text {rep1 } 1}$, the former can be well compensated for by the adaptive sampling method, as shown in Fig. 4(a). The fluctuation of $f_{\text {rep1 }}$ is a remaining factor associated with the fluctuation of the THz comb line position. As the frequency instability of $f_{\text {rep1 }}$ was $\sim 10^{-8}$ from Fig. 3(d), it is expected that the THz comb line fluctuates within a frequency range of $3.3 \mathrm{kHz}$ at $0.33 \mathrm{THz}$ and $5.5 \mathrm{kHz}$ at $0.55 \mathrm{THz}$. Because this $\mathrm{kHz}$ fluctuation is much smaller than the $\mathrm{MHz}$ comb linewidth determined by the inverse of the time window size (4.88 MHz in this article), the influence of the fluctuating $f_{\text {rep1 }}$ is negligible for gapless THz-DCS. $f_{\text {rep1 }}$ and $f_{\text {rep2 }}$ will be tuned with little change in $\Delta f_{\text {rep }}$ by mechanically stretching a portion of the cavity fiber with an additional piezoelectric actuator or motor-driven translation stage. The combination of mode-resolved adaptive sampling THz-DCS with the gapless technique will be studied in a future work.

In summary, we demonstrated the adaptive-sampling nearDoppler-limited $\mathrm{THz}$ comb spectroscopy with the SCDCL. Using the free-running SCDCL with the adaptive sampling method, the long-term instability of the TMF was effectively suppressed, facilitating the long-term acquisition and temporal accumulation of $\mathrm{THz}$ temporal waveforms with a time window extending to multiple laser pulse periods. This results in a broadband, mode-resolved $\mathrm{THz}$ comb spectrum with a frequency sampling spacing of $48.8 \mathrm{MHz}$, a spectral resolution of $4.88 \mathrm{MHz}$, and a power dynamic range of $50 \mathrm{~dB}$. Low-pressure $\mathrm{CH}_{3} \mathrm{CN} /$ air gas with absorption spectral features approaching the $\mathrm{MHz}$ order was measured to be in good agreement with the theoretical predictions. The adaptive sampling scheme could be realized in the analog domain, like this demonstration, or the digital domain by resampling digitized signals. The resolving capability of MHz-level spectral characteristics using a simple SCDCL could greatly expand the applicability of precise $\mathrm{THz}$ spectroscopic techniques to much broader areas of applications.

\section{Acknowledgments}

The work at Tokushima University was supported by grants for the Exploratory Research for Advanced Technology (ERATO) MINOSHIMA Intelligent Optical Synthesizer (IOS) Project (JPMJER1304) from the Japanese Science and Technology Agency; a Grant-in-Aid for Scientific Research (A) (19H00871/ 26246031) from the Ministry of Education, Culture, Sports, Science, and Technology of Japan; and Subsidy for Regional University and Regional Industry Creation, Cabinet Office, 
Japan. The work at Beihang University was supported by NSFC (61435002/61521091/61675014/61675015) and Fundamental Research Funds for the Central Universities. The authors declare no competing financial interests.

\section{References}

1. M. Tonouchi, "Cutting-edge terahertz technology," Nat. Photonics 1, 97-105 (2007).

2. P. U. Jepsen, D. G. Cooke, and M. Koch, "Terahertz spectroscopy and imaging: modern techniques and applications," Laser Photonics Rev. 5, 124-166 (2011).

3. K. Iwaszczuk, H. Heiselberg, and P. U. Jepsen, "Terahertz radar cross section measurements," Opt. Express 18, 26399-26408 (2010).

4. D. H. Auston et al., "Cherenkov radiation from femtosecond optical pulses in electro-optic media," Phys. Rev. Lett. 53, 15551558 (1984).

5. P. Y. Han and X. C. Zhang, "Free-space coherent broadband terahertz time-domain spectroscopy," Meas. Sci. Technol. 12, 17471756 (2001).

6. H. Harde, R. A. Cheville, and D. Grischkowsky, "Terahertz studies of collision-broadened rotational lines," J. Phys. Chem. A 101, 3646-3660 (1997).

7. T. Q. Luong et al., "Onset of hydrogen bonded collective network of water in 1,4-dioxane," J. Phys. Chem. A 115, 14462-14469 (2011).

8. R. J. Falconer and A. G. Markelz, "Terahertz spectroscopic analysis of peptides and proteins," J. Infrared. Millimeters Terahertz Waves 33, 973-988 (2012).

9. I. Coddington, N. Newbury, and W. Swann, "Dual-comb spectroscopy," Optica 3, 414-426 (2016).

10. N. Picqué and T. W. Hänsch, "Frequency comb spectroscopy," Nat. Photonics 13, 146-157 (2019).

11. T. Yasui et al., "Terahertz frequency comb by multifrequencyheterodyning photoconductive detection for high-accuracy, highresolution terahertz spectroscopy," Appl. Phys. Lett. 88, 241104 (2006).

12. T. Yasui et al., "Fiber-based, hybrid terahertz spectrometer using dual fiber combs," Opt. Lett. 35, 1689-1691 (2010).

13. Y. Hsieh et al., "Terahertz comb spectroscopy traceable to microwave frequency standard," IEEE Trans. Terahertz Sci. Technol. 3, 322-330 (2013).

14. G. Villares et al., "Dual-comb spectroscopy based on quantumcascade-laser frequency combs," Nat. Commun. 5, 5192 (2014).

15. Y. Yang et al., "Terahertz multiheterodyne spectroscopy using laser frequency combs," Optica 3, 499-502 (2016).

16. Y. Ren et al., "High-resolution heterodyne spectroscopy using a tunable quantum cascade laser around 3.5 THz," Appl. Phys. Lett. 98, 231109 (2011).

17. J. T. Good et al., "A decade-spanning high-resolution asynchronous optical sampling terahertz time-domain and frequency comb spectrometer," Rev. Sci. Instrum. 86, 103107 (2015).

18. T. Yasui et al., "Enhancement of spectral resolution and accuracy in asynchronous-optical-sampling terahertz time-domain spectroscopy for lowpressure gas-phase analysis," Opt. Express 20, 15071-15078 (2012).

19. X. Zhao et al., "Switchable, dual-wavelength passively modelocked ultrafast fiber laser based on a single-wall carbon nanotube modelocker and intracavity loss tuning," Opt. Express 19, 11681173 (2011).

20. X. Zhao et al., "Polarization-multiplexed, dual-comb all-fiber mode-locked laser," Photonics Res. 6, 853-857 (2018).

21. Y. Liu et al., "Unidirectional, dual-comb lasing under multiple pulse formation mechanisms in a passively mode-locked fiber ring laser," Opt. Express 24, 21392-21398 (2016).

22. T. Ideguchi et al., "Kerr-lens mode-locked bidirectional dual-comb ring laser for broadband dual-comb spectroscopy," Optica 3, 748753 (2016).
23. Q.-F. Yang et al., "Counter-propagating solitons in microresonators," Nat. Photonics 11, 560 (2017).

24. N. B. Hébert et al., "Self-corrected chip-based dual-comb spectrometer," Opt. Express 25, 8168-8179 (2017).

25. S. M. Link et al., "Dual-comb spectroscopy of water vapor with a free-running semiconductor disk laser," Science 356, 1164-1168 (2017).

26. R. M. Li et al., "All-polarization-maintaining dual-wavelength mode-locked fiber laser based on Sagnac loop filter," Opt. Express 26, 28302-28311 (2018).

27. E. Lucas et al., "Spatial multiplexing of soliton microcombs," Nat. Photonics 12, 699-705 (2018).

28. Y. Nakajima, Y. Hata, and K. Minoshima, "High-coherence ultrabroadband bidirectional dual-comb fiber laser," Opt. Express 27, 5931-5944 (2019).

29. X. Zhao et al., "Picometer-resolution dual-comb spectroscopy with a free-running fiber laser," Opt. Express 24, 21833-21845 (2016).

30. S. Mehravar et al., "Real-time dual-comb spectroscopy with a freerunning bidirectionally mode-locked fiber laser," Appl. Phys. Lett. 108, 231104 (2016).

31. R. Liao et al., "Dual-comb spectroscopy with a single freerunning thulium-doped fiber laser," Opt. Express 26, 1104611054 (2018).

32. J. Chen et al., "Dual-comb spectroscopy of methane based on a free-running erbium-doped fiber laser," Opt. Express 27, 11406-11412 (2019).

33. J. Nürnberg et al., "An unstabilized femtosecond semiconductor laser for dual-comb spectroscopy of acetylene," Opt. Express 27, 3190-3199 (2019).

34. G. Hu et al., "Dual terahertz comb spectroscopy with a single freerunning fiber laser," Sci. Rep. 8, 11155 (2018).

35. R. D. Baker et al., "Self-triggered asynchronous optical sampling terahertz spectroscopy using a bidirectional mode-locked fiber laser,' Sci. Rep. 8, 14802 (2018).

36. G. B. Rieker et al., "Frequency-comb-based remote sensing of greenhouse gases over kilometer air paths," Optica 1(5), 290-298 (2014).

37. D. Burghoff, N. Han, and J. H. Shin, "Generalized method for the computational phase correction of arbitrary dual comb signals," Opt. Lett. 44(12), 2966-2969 (2019).

38. L. A. Sterczewski et al., "Computational Doppler-limited dualcomb spectroscopy with a free-running all-fiber laser," $A P L$ Photonics 4, 116102 (2019).

39. T. Yasui et al., "Adaptive sampling dual terahertz comb spectroscopy using dual free-running femtosecond lasers," Sci. Rep. 5, 10786 (2015).

40. J. Roy et al., "Continuous real-time correction and averaging for frequency comb interferometry," Opt. Express 20(20), 21932 (2012).

41. G. Ycas et al., "High-coherence mid-infrared dual-comb spectroscopy spanning 2.6 to $5.2 \mu \mathrm{m}$," Nat. Photonics 12(4), 202-208 (2018).

42. G. Ycas et al., "Mid-infrared dual-comb spectroscopy of volatile organic compounds across long open-air paths," Optica 6(2), 165168 (2019)

43. L. A. Sterczewski, J. Westberg, and G. Wysocki, "Computational coherent averaging for free-running dual-comb spectroscopy," Opt. Express 27(17), 23875-23893 (2019).

44. N. B. Hébert et al., "Self-correction limits in dual-comb interferometry," IEEE J. Quantum Electron. 55(4), 8700311 (2019).

45. C. Janke et al., "Asynchronous optical sampling for high-speed characterization of integrated resonant terahertz sensors," Opt. Lett. 30, 1405-1407 (2005).

46. T. Yasui, E. Saneyoshi, and T. Araki, "Asynchronous optical sampling terahertz time-domain spectroscopy for ultrahigh spectral resolution and rapid data acquisition," Appl. Phys. Lett. 87, 061101 (2005). 
47. H. G. Von Ribbeck et al., "Spectroscopic THz near-field microscope," Opt. Express 16, 3430-3438 (2008).

48. M. Kessler et al., "Microwave spectra and molecular structures of methyl cyanide and methyl isocyanide," Phys. Rev. 79, 54-56 (1950).

49. T. Yasui et al., "Super-resolution discrete Fourier transform spectroscopy beyond time-window size limitation using precisely periodic pulsed radiation," Optica 2, 460-467 (2015).

50. Y. D. Hsieh et al., "Spectrally interleaved, comb-mode-resolved spectroscopy using swept dual terahertz combs," Sci. Rep. 4, 3816 (2014).

51. H. M. Pickett et al., "Submillimeter, millimeter, and microwave spectral line catalog," J. Quant. Spectrosc. Radiat. Transfer 60, 883-890 (1998).

52. A. S. Dudaryonok, N. N. Lavrentieva, and J. V. Buldyreva, " $\mathrm{CH}_{3} \mathrm{CN}$ self-broadening coefficients and their temperature dependences for the Earth and Titan atmospheres," Icarus $\mathbf{2 5 0}$, 76-82 (2015).

53. A. S. Dudaryonok, N. N. Lavrentieva, and J. V. Buldyreva, " $\mathrm{N}_{2}-$ broadening coefficients of $\mathrm{CH}_{3} \mathrm{CN}$ rovibrational lines and their temperature dependence for the Earth and Titan atmospheres," Icarus 256, 30-36 (2015).

54. S. Svanberg, "Differential absorption lidar (DIAL)," in Air Monitoring by Spectroscopic Techniques, M. W. Sigrist, J. D. Winefordner, and I. M. Kolthoff, Eds., Wiley, New York (1994).

Jie Chen received his BS degree in information and computing science from North University of China, Taiyuan, China, in 2012, and his MS degree in signal and information processing from North University of China in 2015. He is currently pursuing his $\mathrm{PhD}$ in optical engineering at Beihang University, Beijing, China. His current research interest includes fiber lasers and their applications.

Kazuki Nitta received his BS degree and his MS degree in engineering from Tokushima University, Japan, in 2018 and 2020, respectively. His research interest includes dual- $\mathrm{THz}$ comb spectroscopy.

Xin Zhao received her BS and PhD degrees from Beihang University, China in 2005 and 2010, respectively. From 2011 to 2012, she was a post-doctoral researcher at Beihang University. Later she was a lecturer and then an associate professor at Beihang. She had been a visiting researcher at National Physical Laboratory, United Kingdom, between 2016 and 2017. Her research interests include ultrafast fiber lasers, optical frequency comb, microresonator combs, and nonlinear optics.

Takahiko Mizuno received his $\mathrm{PhD}$ degree in engineering from Tokushima University, Japan, in 2017. From 2017 to 2019, he was a post-doctoral researcher at Tokushima University, Japan. He is currently an assistant professor at Tokushima University, Japan. His research interests include optical frequency comb and fluorescence spectroscopy.

Takeo Minamikawa received $\mathrm{PhD}$ from Osaka University, Japan, in 2010. From 2011 to 2015, he was engaged in the JSPS Research Fellowship for Young Scientists and served as an assistant professor at Kyoto Prefectural University of Medicine, Japan. Since 2015, he has been an associate professor at Tokushima University. Since 2017, he has also served as PRESTO researcher in Japan Science and Technology Agency (JST), Japan. His research interests include optical-frequencycomb metrology and Raman microscopy for biomedical applications.

Francis Hindle received his $\mathrm{PhD}$ at the University of Manchester, United Kingdom, in 2000. He is currently a professor at the Laboratoire de PhysicoChimie de l'Atmosphère (LPCA), Université du Littoral Côte d'Opale, Dunkerque, France, where he has held a post since 2004. His current research interests include $\mathrm{THz}$ instrumentation and the development of high-resolution spectrometers for gas phase applications.

Zheng Zheng received his BEng degree from Tsinghua University, China, and MSEE and PhD degrees from Purdue University, United States. He was with Lucent Technologies before joining Beihang University as a professor. He has authored more than 230 journal and conference papers, and holds six US patents and more than 20 Chinese patents. His current research interests include ultrafast and nonlinear optics, microwave photonics, and nanophotonics. He is a fellow of the Chinese Institute of Electronics.

Takeshi Yasui received his first $\mathrm{PhD}$ degree in engineering from Tokushima University, Japan, in 1997, and his second PhD degree in medical science from Nara Medical University, Japan, in 2013. After post-doctoral research at the National Research Laboratory of Metrology, Japan, and a period as an assistant professor at Osaka University, he is currently a professor at the Institute of Post-LED Photonics, Tokushima University, Japan. His research interests include $\mathrm{THz}$ and optical combs and second-harmonic-generation microscopy. 